\title{
Aspectos clínicos e socioeconômicos das dislipidemias em portadores de doenças cardiovasculares
}

I 1 Ana Paula Zambuzi Cardoso, 2 Maria Suely Nogueira,

${ }^{3}$ Miyeko Hayashida, ${ }^{4}$ Luiz de Souza, ${ }^{5}$ Evandro José Cesarino I

Resumo: O presente artigo aborda os aspectos clínicos e socioeconômicos decorrentes da presença de dislipidemias em portadores de doenças cardiovasculares (DCV). Existem inúmeros estudos relacionados às DCV, uso de hipolipemiantes orais como as estatinas, e os aspectos econômicos envolvidos com impacto na área da saúde. Além de evidenciar a importância do tratamento das dislipidemias, o artigo busca demonstrar o ponto de vista farmacoeconômico, ou seja, dos custos gerados com o tratamento farmacológico desta patologia versus os custos decorrentes dos eventos cardiovasculares acometidos e suas consequências. Existe, portanto, relevante relação entre os impactos sociais decorrentes de incapacidade física e laborativa, aposentadorias precoces, entre outros custos importantes que poderiam ser evitados com uma análise econômica abrangente e eficiente realizada nos serviços de saúde do Brasil. Neste contexto, é enfatizada a importância da análise conjunta dos aspectos clínicos e socioeconômicos das dislipidemias que poderiam influenciar nas decisōes das autoridades de saúde no momento da elaboração de protocolos clínicos de tratamentos farmacológicos a serem implementados no SUS.

> Palavras-chave: doenças cardiovasculares; dislipidemias; estatinas; economia em saúde.
1 Farmacêutica, mestranda em Biociências Aplicadas à Farmácia pela Faculdade de Ciências Farmacêuticas de Ribeirão Preto (FCFRP-USP). Endereço eletrônico: ana.paula.cardoso@ usp.br

2 Enfermeira, livre-docente em Enfermagem pela Escola de Enfermagem de Ribeirão Preto (USP) Endereço eletrônico: msnog@eerp.usp.br

\author{
3 Enfermeira, doutora em \\ Enfermagem Fundamental \\ pela Escola de Enfermagem de \\ Ribeirão Preto (USP). Endereço \\ eletrônico: miyeko@eerp.usp.br \\ 4 Estatístico, professor doutor \\ aposentado da Faculdade de \\ Medicina de Ribeirão Preto \\ (USP). Endereço eletrônico: \\ Idsouza@fmrp.usp.br \\ 5 Médico cardiologista, \\ professor do Departamento de \\ Análises Clínicas Toxicológicas e \\ Bromatológicas da FCFRP-USP, \\ doutor em Saúde Preventiva \\ pela FMRP-USP. Pesquisador \\ CNPq. Endereço eletrônico: \\ cesarino@fcfrp.usp.br
}

Recebido em: 19/02/2011 Aprovado em: 22/04/2011. 


\section{Introdução: dados epidemiológicos das doenças cardiovasculares}

As doenças cardiovasculares (DCVs) constituem a principal causa de óbito em importante parcela da população adulta, particularmente aquela maior de 30 anos de idade na maioria dos países do Ocidente. Tal posição tem sido mantida, apesar do declínio acentuado da mortalidade cardiovascular observada nos países desenvolvidos, nas últimas décadas (BEAGLEHOLE, 1990).

Até meados do século XX, a idade era considerada o maior fator determinante da ocorrência das DCVs, em especial da doença arterial coronária (DC). Estudos posteriores mostraram que pacientes sobreviventes de infarto agudo do miocárdio (IAM) apresentavam níveis séricos mais elevados de colesterol total (CT), quando comparados a pacientes sadios (XAVIER, 2004).

A despeito do grande avanço científico ocorrido nas últimas décadas, na prevenção e no tratamento das DCVs, estima-se que estas serão responsáveis por $40 \%$ de todas as mortes ocorridas no mundo em 2020, o dobro daquelas esperadas devido ao câncer, com uma estimativa de 11,11 milhões de mortes (MURRAY; LOPEZ, 1996).

Nos EUA, desde 1984 morrem mais mulheres devido a causas cardiovasculares do que homens. Em 2001, cerca de 500 mil mulheres e 430 mil homens foram a óbito por DCV nesse país. As razões para explicar este fato incluem a maior expectativa de vida feminina, a menor taxa de mortes por violência e a maior frequência de complicações cardiovasculares entres as mulheres (AHA, 2004).

Nos últimos 30 anos, ocorreu um declínio da mortalidade por DCV em países desenvolvidos, enquanto elevações relativamente rápidas e substanciais têm ocorrido em países em desenvolvimento, dentre os quais o Brasil. De acordo com as projeções da Organização Mundial da Saúde (OMS), esta elevação das DCVs tende a persistir, agravando o quadro de morbimortalidade elevadas nesses países (SBC, 2007).

Em relação ao Brasil, a prevalência das DCVs varia de acordo com as regiões brasileiras. Nos estados do Sul e Sudeste, por exemplo, observou-se maior percentual de óbitos por causa cardiovascular, 33,5\% e 34,9\%, respectivamente. Em 1930, a percentagem de causa mortis por doenças do aparelho circulatório (DAC) no Brasil era de 12\%; entretanto, em 1999 essa percentagem triplicou, 
chegando a 32\% (BRASIL, 2002a). Este fato provavelmente pode ser atribuído à

elevada prevalência de fatores de risco modificáveis e tratáveis para a aterosclerose, tais como as dislipidemias (FONSECA, 2003).

No Brasil, em 1998, o principal grupo de doenças que acarretaram internações de idosos com 60 anos ou mais de idade foi a DAC, sendo a insuficiência cardíaca (IC) a principal causa básica de internação hospitalar nesta faixa etária (BRASIL, 2002b).

No ano de 2005, a mortalidade por DAC na cidade de São Paulo-SP situou-se em 32,3\%, sendo que de um total de 100.000 habitantes, houve ocorrência de IAM e de DCV em 42,1\% e 49,4\%, respectivamente (BRASIL, 2007).

Já com relação a 2006, a percentagem de internações por DAC no Brasil foi de $10,11 \%$, equivalente a 1.146 .867 internações. Só a cidade de São PauloSP foi responsável por 56.463 internações por DAC, sendo que 77,98\% desses indivíduos se situavam na faixa etária acima de 50 anos (DATASUS, 2008).

\section{Dislipidemias e suas consequências}

As dislipidemias consistem em modificações nos níveis lipídicos na circulação, caracterizando qualquer alteração envolvendo o metabolismo lipídico, sendo classificadas em primárias (origem genética) ou secundárias (doenças, estilos de vida, medicamentos, entre outros). O maior impacto das dislipidemias nas DCVs se deve às hiperlipidemias. As dislipidemias, em especial as hiperlipidemias, causam alterações do sistema de hemostasia, aumentando a formação da placa aterosclerótica, quer induzindo a formação de trombos que irão ocluir as artérias, interrompendo o fluxo sanguíneo e causando morte tecidual (MILLER, 1994).

De acordo com a Sociedade Brasileira de Cardiologia (SBC), 40\% dos brasileiros têm CT $\geq 200 \mathrm{mg} / \mathrm{dl}$ e 13\% têm CT $\geq 240 \mathrm{mg} / \mathrm{dl}$ (MARTINEZ, 2003).

A aterosclerose é uma doença inflamatória crônica dependente de diversos fatores e é resposta à agressão endotelial, afetando artérias de médio e grande calibre. Esta patologia era considerada uma doença degenerativa, de progressão lenta e apresentando sintomas decorrentes de interferências mecânicas no fluxo sanguíneo. Contudo, pesquisas recentes têm mostrado que os componentes celulares e moleculares do desenvolvimento e da progressão da aterosclerose parecem ser um processo dinâmico, inflamatório e modificável (MARTINEZ, 2003).

Os processos ateroscleróticos, incluídos nas DACs, têm como principais manifestações as cerebrovasculares, coronárias e insuficiência cardíaca (IC). 
Quando acometem as artérias coronárias, podem provocar, por exemplo, IAM, angina pectoris e IC; já o acometimento no território cerebral pode ocasionar derrames ou acidente vascular encefálico (AVE) e aneurismas; nas artérias dos membros inferiores, podem ocasionar dores e gangrenas; nos intestinos, colites isquêmicas; nos órgãos genitais masculinos, podem gerar impotência; e nas grandes artérias, pode haver dilatação da aorta como aneurismas no tórax ou no abdome (MION JR; NOBRE, 2002).

Dentre as enfermidades cardiovasculares, a doença isquêmica cardíaca (DIC) e as doenças cerebrovasculares (DCBs) são as mais frequentes (CHOR et al., 1995).

Em 1998, as três principais causas de óbitos no Brasil foram a DCBs, seguida da DIC e o IAM, acarretando 217.150 mortes. Em 2000, a IC ficou na quinta posição dentre as causas mais frequentes de internação, com 398.489 internações no país. Esta situação clinica se situou também na quinta posição dentre as causas responsáveis pelos maiores gastos globais com internações do Sistema Único de Saúde - SUS (BRASIL, 2002b).

$\mathrm{O}$ estudo de Framingham demonstrou que, enquanto na população masculina, a DIC predomina na meia idade, na feminina predomina após a menopausa, passando a ser a principal causa de morte nas idades acima de 50 anos (LERNER; KANNEL, 1986). Calcula-se que 0,2\% da população mundial seja hospitalizada todos os anos, principalmente para o tratamento da IC, e o volume de internações deste tipo está aumentando significativamente (McMURRAY, 1993; GILLUM, 1993; ANDERSON; WAGSTEIN, 1993).

No Reino Unido, a IC responde por pelo menos 5\% de todas as internaçôes de medicina e geriatria, superando o número de pacientes admitidos com IAM e angina instável (PARAMESHWAR et al., 1992; McMURRAY, 1993). A duração média da hospitalização por IC no Reino Unido é de duas semanas; nos EUA, a hospitalização é menos demorada (McMURRAY, 1993; GILLUM, 1993).

Estima-se que, em relação à população geral, a IC determine uma redução na expectativa de vida de 6,7 anos nos homens e de 5,1 nas mulheres por 1.000 pessoas (STEWARD, 2001).

O AVE é um problema de saúde mundial em ambos os sexos, com incidência de 600 mil casos novos ou recorrentes ao ano. O AVE responde por uma em cada 14 mortes nos EUA, sendo a terceira causa de morte entre as DCVs (AHA, 2002). 
E, embora tenha havido declínio na mortalidade por AVE nos EUA, o número de mortes atribuíveis a esta moléstia tende a se elevar por um desnível entre o declínio da mortalidade por AVE e o envelhecimento da população (NHLBA, 2002).

A ruptura de aneurisma de aorta abdominal ocupa o décimo lugar entre as causas de morte em homens acima de 55 anos, porém quando diagnosticado precocemente, a mortalidade cai para 5\% ou menos. À medida que a população envelhece, a diferença entre a incidência de aneurismas de aorta entre homens e mulheres tende a diminuir (GAGLIARDI; ROMITI, 2005).

A análise da morbidade hospitalar por DAC de 1987 a 1992 em Ribeirão Preto-SP revela-nos que o número médio de internações anuais por DAC foi de 10.116, número médio de óbitos anuais por DAC foi de 841 (8,4\% das internações por DAC) e a IC foi a principal causa de hospitalização por DAC (16,3\% das internaçōes por DAC) (CESARINO; VICHI, 1995).

No ano de 1995, as DACs representaram 30,8\% dos óbitos entre pessoas residentes em Ribeirão Preto-SP, ocorrendo 199 óbitos devidos a IAM (6,5\%), constituindo a segunda principal causa básica de óbitos entre pessoas residentes em Ribeirão Preto-SP (RIBEIRÃO PRETO, 1996). Neste mesmo ano, ocorreram 18.766 internações hospitalares, tendo como diagnóstico principal as DACs (10,98\% do total das internações) em hospitais de Ribeirão Preto-SP e região, resultando em 1.312 óbitos (7\%) (USP, 1999).

Podemos citar os indicadores de magnitude da hiperlipidemia como problema social, tais como sua mortalidade devido à elevada prevalência; mortalidade decorrente de suas complicações como causas básicas ou associadas; custos diretos com morbidade como internaçôes, demanda ambulatorial e custos globais com tratamento, controle e reabilitação; custos sociais: taxa de absenteísmo ao trabalho, benefícios sociais (auxílio-doença, pensões e aposentadoria por doença) e anos de vida produtiva perdidos; e por fim, as repercussóes sociais como qualidade de vida de pacientes e famílias, além de afetar a produtividade e o crescimento econômico do país (BRASIL, 1993a).

A implementação de programas educativos permanentes voltados para o esclarecimento dos fatores de risco cardiovasculares junto à população poderá, no longo prazo, ter efeitos favoráveis na redução da morbimortalidade por DCV (CESARINO et al., 1997). 


\section{Impacto da redução do perfil lipídico na prevenção primária e secundária das doenças cardiovasculares}

Os inibidores da HMG-CoA redutase, uma das enzimas-chave na síntese intracelular do colesterol, também conhecidas como estatinas, atuam reduzindo o conteúdo intracelular de colesterol; como consequência, ocorre um aumento do número de receptores de LDL nos hepatócitos, que então removem maior quantidade de VLDL, IDL e LDL da circulação para repor o colesterol intracelular (SBC, 2007).

As estatinas possuem, além de atuação no perfil lipídico, efeitos pleiotrópicos ou independentes do colesterol, tais como: inibem a proliferação das células do músculo liso e a migração induzida por fatores de crescimento, o que influencia a estabilidade da placa e na aterotrombose; melhoram a função endotelial, pelo aumento e disponibilidade do óxido nítrico, interferindo, assim, no processo patológico da aterosclerose (MENNICKENT et al., 2008).

Com a descoberta e o desenvolvimento das estatinas, em pouco tempo vários estudos foram publicados durante os anos 90 , demonstrando que elas poderiam reduzir a morte por DAC e ataques do coração em 20-50\%, dependendo do nível de colesterol sanguíneo e da presença ou ausência de outros fatores de risco para DCV (FREEMAN, 2006).

Estudo britânico revela que pacientes com DIC que fazem uso de estatinas apresentam uma redução de $39 \%$ de risco de morte em relação a pacientes que não realizam tratamento com estes fármacos (HIPPISLEY-COX, 2006).

Importantes estudos clínicos em pacientes com manifestação de DAC foram realizados. O estudo 4S (Scandinavian Simvastatin Survival Study) demonstrou, no grupo tratado com sinvastatina (SINV, 20 a $40 \mathrm{mg} / \mathrm{dia}$ ) reduções de $25 \%$ nos níveis de CT, 35\% nos níveis de LDL-colesterol (LDL-C) e aumento de 8\% nos níveis de HDL-colesterol (HDL-C). A redução de eventos coronarianos foi de $34 \%$ e a mortalidade deste grupo por DAC foi reduzida em $42 \%$. A diminuição do número de pacientes que necessitaram de hospitalização e de dias de internação também foi acompanhada por diminuição de gastos. No 4S, por exemplo, houve $32 \%$ de redução nos custos hospitalares por ano de sobrevida (SSSSG, 1994).

Além do 4S, outros quatro grandes estudos clínicos com estatinas foram realizados, CARE (Cholesterol and Recurrent Events Trial), LIPID (LongTerm Intervention with Pravastatin in Ischaemic Disease Study), WOSCOPS 
(West of Scotland Coronary Prevention Study) e AFCAPS/TexCAPS (Air

Force/Texas Coronary Atherosclerosis Prevention) que avaliaram pacientes de alto risco para eventos cardiovasculares e/ou portadores de eventos prévios. (SHEPHERD et al., 1995, SACKS et al., 1996; THE LONG-TERM..., 1998; DOWNS et al., 1998).

O HPS (The Heart Protection Study), um dos maiores estudos clínicos de prevenção secundária controlado por placebo realizado com uma estatina, demonstrou que a SINV administrada a pacientes de alto risco levou a uma redução de 30\% no LDL-C, que foi associada com reduções de 30 a 40\% em todos os eventos cardiovasculares e DC (HPSCG, 2002).

Já o estudo CARDS (Collaborative Atorvastatin Diabetes Study) avaliou pacientes diabéticos em prevenção primária da DC, observando, nos pacientes que usaram atorvastatina (ATORV), redução de $26 \%$ do CT e em $40 \%$ os níveis de LDL-C, acompanhando-se de redução de 37\% no desfecho composto (cardiovasculares somados a AVE), de 36\% nas síndromes isquêmicas agudas e em 48\% nos AVEs (COLHOUN, 2004).

O estudo GREACE (Greek Atorvastatin and Coronary-heart-disease Evaluation) revelou que o tratamento intensivo de redução de lipídios com ATORV, na dose de até $80 \mathrm{mg} /$ dia em pacientes com DC estabelecida, foi mais efetivo na redução da morbimortalidade por DC que os cuidados habituais utilizados na prática clínica (ATHYROS, 2002). O mesmo fato foi observado no estudo ALLIANCE, onde $72,4 \%$ dos pacientes tratados com ATORV atingiram a meta de LDL-C $<100 \mathrm{mg} /$ $\mathrm{dl}$ contra $40 \%$ daqueles tratados com os cuidados habituais (KOREN, 2004).

$\mathrm{Na}$ Alemanha, foi realizado um dos mais importantes estudos observacionais, o PROCAM (Prospective Cardiovascular Munster), demonstrando que as diferenças de LDL-C são mais expressivas entre os pacientes com DC prematura. Entre os idosos que desenvolvem IAM, cresce a importância dos níveis de HDL (ASSMANN, 2004).

A prevalência de hipertrigliceridemia é relativamente frequente na população em geral. $\mathrm{Na}$ população americana baseada no Third National Health and Nutrition Examination Survey, 30\% dos 8.814 participantes do estudo com idade superior a 20 anos tinham níveis séricos de triglicérides (TG) $>150 \mathrm{mg} / \mathrm{dL}$. A prevalência de níveis elevados de TG foi de 42,8\% em pessoas com mais de 50 anos (ALEXANDER, 2003). 


\section{Aspectos socioeconômicos das doenças cardiovasculares}

As DCVs quase sempre repercutem nas atividades laborativas e produtivas do homem, podendo ser caracterizadas como doenças ocupacionais ou relacionadas ao trabalho, o que geralmente não se observa na literatura médica, mas acabam, assim mesmo, acarretando implicações em termos econômicos e sociais no sistema previdenciário.

O custo social das consequências das hiperlipidemias deve ser entendido numa perspectiva cumulativa, ou seja, as pensões, as aposentadorias por doença e os auxíliosdoença acumulam-se no decorrer do tempo, tornando o ônus sobre a sociedade muito maior do que as estimativas apresentadas para cada item em separado.

As DCVs são responsáveis por significativos casos de incapacidade, contribuindo para o absenteísmo laboral (BRASIL, 1993b). O absenteísmo ao trabalho deve ser lembrado como outro dos custos mensuráveis das consequências das hiperlipidemias e que afeta diretamente a produtividade do país.

Em relação à mortalidade precoce (15-59 anos), foram estimados, para 1985, cerca de 290.000 anos de vida produtiva perdidos no Brasil por mortalidade pelo AVE, e uma redução significativa desse total poderia ocorrer, caso a hiperlipidemia fosse precocemente detectada, tratada e controlada (LESSA, 1990). Seriam mortes evitáveis, do mesmo modo que seria evitável uma grande parcela dos anos de vida economicamente produtivos perdidos em razão de incapacidade permanente, resultando em aposentadorias prematuras por invalidez e um enorme ônus para a sociedade.

Sem tratamento oportuno e adequado, a hiperlipidemia contribui para a instalação e o progressivo agravamento das DCVs, tornando-se um grave problema de saúde pública, também em termos de custos materiais, pois consome recursos econômicos tanto em relação a despesas de terapêutica clínica como de tratamento sob regime de internação hospitalar, sobretudo por procedimentos intervencionistas, tais como angioplastia transluminal coronária com ou sem implante de Stent e cirurgia de revascularização miocárdica.

A aposentadoria precoce leva a uma redução da renda familiar, interferindo negativamente na qualidade de vida do paciente e de sua família. Esses dados revelam a importância social e econômica das DCVs, particularmente as consequências da hiperlipidemia, como principais causas geradoras de obtenção 
de benefícios previdenciários. Isto acarreta um grande prejuízo econômico sofrido pela nação, em decorrência da eliminação antecipada da força de trabalho dos cardiopatas, excluindo gastos com assistência médico-hospitalar.

As consequências diretas e indiretas da hiperlipidemia não diagnosticada ou mal controlada poderiam acarretar desemprego, incapacidade física e laborativa, custos médico-hospitalares e aposentadoria precoce. Estes impactos sociais e econômicos precisam ser bem analisados, pois são exatamente eles que muitas vezes dão o substrato para tomada de decisões administrativas e políticas no campo da Saúde. Neste sentido, um investimento em ações preventivas poderá resultar na Previdência Social brasileira, em médio e longo prazo, em significante benefício social e redução substancial de custos, engajando-se na profilaxia ofensiva contra este verdadeiro flagelo social que são as DCVs, influenciadas significativamente pela hiperlipidemia.

Os responsáveis por provimento de programas de saúde enfrentam o dilema de substituir ou não medicamentos já em uso, e de adicionar ou não novas drogas a esquemas terapêuticos tradicionais, buscando obter uma relação custo/ benefício socialmente adequada. Com essas premissas, a otimização da aplicação dos limitados recursos destinados à saúde poderia ser obtida com a utilização dos preceitos da Medicina Baseada em Evidências, que usualmente tem sido implementada em protocolos de novos e antigos procedimentos e medicamentos de alto custo (BOCCHI; MARIN-NETO, 2001).

Acometendo grande parcela da população, a DIC resulta em substancial custo de tratamento na fase hospitalar devido a intervenções e procedimentos invasivos destinados à detecção de complicaçooes e avaliação da terapêutica. Além disso, há necessidade do cuidado contínuo de muitos pacientes, geralmente incapacitados em definitivo para suas atividades pessoais e profissionais, que apresentam alta taxa de recorrência de eventos, gerando gastos consideráveis para o SUS.

Sabe-se que pode ocorrer IAM sem hiperlipidemia, bem como existem outras etiologias para a gênese da IC, além da isquêmica; entretanto, esses custos não estão incluindo os gastos com as internações clínicas com o diagnóstico principal (AVE), que também poderia ter a aterosclerose envolvida, além de custos com o sistema produtivo (absenteísmo) e previdenciário (aposentadorias precoces e outros benefícios previdenciários). 


\section{Avaliação econômica em saúde: uma abordagem da farmacoeconomia}

A economia da saúde, utilizando-se de instrumentos de avaliação, concilia necessidades de ordem técnica, resultados clínicos e custos de intervenções terapêuticas, com o objetivo de identificar, entre duas ou mais alternativas, aquela mais adequada a um grupo de indivíduos, instituição e sociedade (FOLLADOR, 1999).

A economia analisa os custos, benefícios e a melhor maneira de distribuir os recursos, utilizando-se de metodologias que podem ser aplicadas em situaçôes em que são necessárias decisões e há escassez ou limitação de recursos. Esta definição se aplica perfeitamente ao setor da saúde, em que os recursos são geralmente insuficientes e limitados, e a decisão de como utilizá-los é quase sempre centralizada nas figuras dos profissionais da saúde, administradores ou políticos (DEL NERO, 1995).

A farmacoeconomia é um conjunto de atividades dedicadas, de modo geral, à análise econômica na área da Assistência Farmacêutica, como a gestão de serviços farmacêuticos, avaliação da prática profissional e avaliação econômica de medicamentos; de modo específico, à descrição e à análise dos custos e das consequências da farmacoterapia para o paciente, o sistema e a sociedade (BRASIL, 2009).

$\mathrm{Na}$ avaliação econômica global de um medicamento, distingue-se a avaliação clínica, baseada na eficácia ou efetividade, e a avaliação farmacoeconômica, baseada na eficiência, em que se inclui o cálculo de custos. Desta forma, qualquer método que traga informaçóes sobre custos e efeitos de um medicamento pode ser utilizado como base para a realização de uma avaliação farmacoeconômica (SACRISTÁN DEL CASTILHO, 1995).

A farmacoeconomia representa um instrumento de apoio para tomada de decisões, que envolvem avaliação e direcionamento de investimentos baseados numa distribuição mais racional de recursos, permitindo aos profissionais conciliarem necessidades terapêuticas com possibilidades de custeio individual, das empresas provedoras de serviços ou de sistemas de saúde. Assim sendo, a introdução dos estudos farmacoeconômicos, no cenário da saúde, tem permitido incorporar um novo critério - o econômico - na escolha de alternativas terapêuticas (SECOLI, 2005). 
Por utilizar terminologia técnica específica, a condução dos estudos farmacoeconômicos (EF) demanda a exposição conceitual de pontos consensuais relativos a custos, outcomes, eficácia, efetividade e eficiência.

O custo representa o valor de todos os insumos (trabalho, materiais, pessoal, entre outros) utilizados na produção e distribuição de bens ou serviço (BOMBARDIER; EISENBERG, 1985). Ele representa o elemento comum dos métodos da farmacoeconomia, que engloba os recursos considerados relevantes na aplicação do tratamento. Os custos podem ser classificados em direto, indireto e intangível (VILLAR, 1995). Esta classificação permite a constituição do custo total de um produto, programa ou serviço.

Custos diretos são aqueles relacionados diretamente aos serviços de saúde, que implicam dispêndios imediatos, de identificação objetiva, correspondendo aos cuidados médicos e não médicos (BOMBARDIER; EISENBERG, 1985). Os custos diretos relativos aos cuidados médicos contemplam produtos e serviços desenvolvidos para prevenir, detectar e/ou tratar uma doença - por exemplo, os honorários profissionais. Os custos diretos não-médicos são decorrentes da doença, resultam do processo de adoecimento, mas não envolvem os serviços médicos - por exemplo, custo de alimentação, transporte, residência temporária, entre outros.

Os custos indiretos são relacionados à perda da capacidade produtiva do indivíduo ante o processo de adoecimento ou mortalidade precoce. Eles representam dias de trabalho perdidos, incapacidade de realizar as atividades profissionais, tempo gasto em viagens para receber cuidado médico e morte prematura decorrente da doença (BOMBARDIER; EISENBERG, 1985; VILLAR, 1995).

Os intangíveis são custos de difícil mensuração monetária. Embora muito importantes para os pacientes, ainda necessitam de significado econômico. São os custos do sofrimento, da redução da qualidade de vida, entre outros (BOMBARDIER; EISENBERG, 1985; VILLAR, 1995).

A análise de custo-efetividade representa o tipo de análise mais utilizado na farmacoeconomia, porque permitea o uso na prática cotidiana das mesmas unidades utilizadas nos ensaios clínicos. No conjunto dos fatores relacionados ao aumento dos custos com saúde, evidenciam-se as transformações nos padrões de morbimortalidade das sociedades contemporâneas, que podem ser explicadas pela redução das doenças infectocontagiosas e o aumento das doenças crônicodegenerativas, que estão, em grande parte, relacionadas a fatores de risco 
(tabagismo, alimentação, estresse, sedentarismo, entre outros) decorrentes do processo de modernização dessas sociedades.

Essas mudanças epidemiológicas afetaram sensivelmente o panorama dos gastos com o setor da saúde, pois enquanto as primeiras demandam predominantemente medidas preventivas, as outras exigem assistência secundária ou terciária, envolvendo tecnologia médica de alto custo. Esta última, expressa por exames diagnósticos e complementares e equipamentos sofisticados, bem como o incremento da indústria farmacêutica, proporcionou melhoria da qualidade dos serviços de saúde, mas elevou sensivelmente seus custos, pois incorporou, além do capital investido (equipamentos), recursos humanos qualificados para operar e interpretar as novas tecnologias (MÉDICI, 1994; BARRETO; CARMO, 1998).

\section{Análise de custos do tratamento das dislipidemias}

É sabido que as dislipidemias, na maioria das vezes, não provocam sintomas, e os indivíduos portadores deste distúrbio metabólico acabam não procurando o SUS com esta finalidade específica. A estratificação de risco deve continuar sendo utilizada para discernir os pacientes que poderiam mais se beneficiar com a utilização desta medicação, que são aqueles com manifestações clínicas de DIC. Estes apresentam risco elevado de desenvolver um evento coronariano em dez anos, ou seja, acima de 20\%, de acordo com o Escore de Risco de Framingham, mesmo sendo assintomáticos (LERNER; KANNEL, 1986; WILSON et al., 1998; WOOD et al., 1998).

Um estudo farmacoeconômico espanhol avaliou várias estatinas (ATORV, fluvastatina (FLUV), SINV e pravastatina (PRAV) em pacientes com hipercolesterolemia e com um ou mais fatores de risco cardiovasculares durante seis meses. Como resultado, observou-se maior relevância clínica para a ATORV e a mesma se mostrou mais efetiva e menos custosa que a PRAV. Quando comparada a FLUV e SINV, houve um custo adicional por paciente, daí a necessidade de um profissional tomador de decisão para avaliar os gastos e decidir pela melhor situação (TARRAGA-LOPEZ, 2005).

Outro estudo avaliou indivíduos diabéticos tipo-2 e com LDL acima de $100 \mathrm{mg} / \mathrm{dL}$, sem história prévia de IAM, angina pectoris, na prevenção primária de eventos coronários graves, utilizando cinco estatinas: SINV, PRAV, ATOR, FLUV e lovastatina (LOV). A ATORV reduziu em 39\% os níveis de LDL 
elevado, seguida da SINV, que reduziu em 33\%. Numa análise econômica realizada, a ATORV apresentou maiores custos; entretanto, maior efetividade clínica (BRANDLE, 2003).

Em virtude de as DCV constituírem importante problema de saúde pública, os novos estudos de custo-efetividade em farmacoeconomia e a necessidade de uma conscientização de prevenção vêm progressivamente aumentando no mundo todo (FONSECA, 2003). Segundo dados do Instituto Brasileiro Geográfico e Estatístico (IBGE), a população brasileira estimada em 2004 foi de 181.581.024, sendo que, destes, 107.092.599 são considerados adultos (maiores de 20 anos). No Estado de São Paulo, residiam 39.239.362 pessoas, dos quais 25.028.594 eram considerados adultos.

Dados levantados na Campanha Nacional contra o Colesterol de 2002 revelam que $13 \%$ da população adulta brasileira apresentam níveis de CT maior que 240 $\mathrm{mg} / \mathrm{dl}$, o que corresponderia hoje a aproximadamente 14 milhões de pessoas com níveis elevados de CT - indivíduos em potencial para tratamento com estatinas. No Estado de São Paulo, esse número chegaria a 3,2 milhões de pacientes (MARTINEZ et al., 2003). Segundo dados do DATASUS, cada comprimido de SINV de 20mg adquirido pelo SUS por processo licitatório apresenta o preço mínimo de R \$ 0,07 (sete centavos de real) no Estado de São Paulo, que seria a estatina de menor custo existente no mercado (DATASUS, 2010).

Considerando que a posologia geralmente indicada seria de um comprimido de $20 \mathrm{mg}$ ao dia, cada paciente com hipercolesterolemia acarretaria um gasto mensal de $\mathrm{R}$ \$ 2,10 (dois reais e dez centavos) e anual de $\mathrm{R} \$ 25,20$ (vinte e cinco reais e vinte centavos). Isto geraria o montante de $\mathrm{R} \$ 80$ milhôes e 640 mil de reais gastos anuais com pacientes do Estado de São Paulo, que seriam passíveis de utilização de estatinas (MARTINEZ et al., 2003; IBGE, 2008; GUIA FARMACÊUTICO BRASÍNDICE, 2010). Entretanto há relato na literatura de melhor custo-efetividade com a utilização da ATORV $10 \mathrm{mg}$, também disponível pelo SUS, através do Programa de Dispensação de Medicamentos Excepcionais do Ministério da Saúde, mesmo com o custo de R \$ 1,50 por comprimido,segundo dados do DATASUS (FONSECA, 2003; DATASUS, 2010).

Uma das iniciativas tomadas pelo governo com foco na redução de custos com alguns medicamentos foi a criação do medicamento genérico, há mais de dez anos, e que atualmente é conhecida por $90 \%$ da população brasileira. Com 
este programa, os pacientes com dislipidemias que gastariam no ano de 2000 uma média de $\mathrm{R} \$ 3.675,36$ comprando o medicamento de referência, sendo que, em 2001, com o genérico correspondente (SINV), desembolsariam R\$ 1.634,40, representando uma economia de 55,53\% (GUIA FARMACÊUTICO BRASÍNDICE, 2010).

Um avanço recente obtido pela comunidade foi a padronização da SINV no Programa Farmácia Popular do Governo Federal, ocorrido no dia 26/04/10, a um custo bastante acessível: cada comprimido de $20 \mathrm{mg}$ custa $\mathrm{R} \$ 0,25$ (vinte e cinco centavos de real), ampliando o acesso do medicamento para população (DOU, 27/04/10).

\section{Considerações finais}

Infelizmente, uma grande parcela da população ainda não é tratada de acordo com as diretrizes sobre dislipidemias para a prevenção de aterosclerose, que em geral traduzem os resultados dos melhores ensaios clínicos e recomendações para maiores benefícios a esta população.

A instituição de saúde que trilhar pelo caminho de analisar os aspectos clínicos e socioeconômicos do tratamento das dislipidemias com certeza atingirá o propósito procurado por todos, que é obter êxitos financeiros e sociais. De posse dos argumentos apresentados, é interessante que sejam realizadas pesquisas que analisem mais precisamente custos com prevenção e tratamento, não só desta patologia como também de outras. A determinação da eficácia de tratamentos e procedimentos é de fundamental relevância, pois os recursos econômicos são limitados e, assim, as prioridades sociais devem ser estabelecidas.

Hoje em dia, não podemos dissociar os nossos atos do contexto socioeconômico dos pacientes e do sistema em que estamos inseridos. Numa análise descritiva de custos, observa-se apenas o lado de uma moeda: o custo do tratamento. Para a sociedade como um todo, é fundamental se avaliar também o custo do nãotratamento da doença, ou seja, ocorrência de mais eventos, mais internaçóes, mais procedimentos, maior sofrimento e incapacidade de pacientes e mais mortes.

Somente estudos farmacoeconômicos completos poderiam ajudar a avaliar, a partir de que nível de risco de DIC, o tratamento com hipolipemiantes tornarse-ia custo-efetivo dentro da realidade atual. Os diversos estudos de prevenção primária e secundária com a utilização das estatinas demonstraram, além da 
redução da morbimortalidade cardiovascular, a redução da necessidade de procedimentos como angioplastias ou cirurgias de revascularização miocárdica. Tal fato deve ser levado em consideração na administração de recursos pelo SUS, pois a economia imediata com a limitação do fornecimento da medicação à população que teria indicação da utilização deste fármaco poderia ser menor que uma economia futura com a realização de procedimentos de alto custo e internaçôes hospitalares, que poderiam ser evitadas.

Neste sentido, justifica-se a realização de estudos mais aprofundados que analisem do ponto de vista econômico e também da influência acarretada pelo uso contínuo de estatinas em indivíduos portadores de dislipidemias, assim como seu impacto em relação à morbimortalidade cardiovascular.

\section{Referências}

ALEXANDER, C.M. et al. NCEP defined metabolic syndrome, diabetes, and prevalence of coronary heart disease among NHANES III participants age 50 years and older. Diabetes, Alexandria, v. 52, n.5, p.1210-14, 2003.

AMERICAN HEART ASSOCIATION. Heart Disease and Stroke Statistics. 2003 Update. Dallas, 2002.

AMERICAN HEART ASSOCIATION. Heart Disease and Stroke Statistics. Dallas, 2004. ANDERSON, B.; WAGSTEIN, F. Spectrum and outcome of congestive heart failure in a hospitalized population. American Heart Journal, Durham, v. 126, n.3, p. 632-40, 1993. ASSMANN, G. Prospective Cardiovascular Munster (PROCAM). The British Journal of Cardiology, Londres, v. 11, p. S1-S2, 2004.

ATHYROS, V.G. et al. Treatment with atorvastatin to the National Cholesterol Educational Program goal versus "usual" care in secondary coronary heart disease prevention: GREek Atorvastatin and Coronary-heart-disease Evaluation (GREACE) study. Currenty Medical Research \& Opinion, Nova York, v. 18, n. 4, p. 215-9, 2002.

BEAGLEHOLE, R. International trends in coronary heart disease mortality, morbidity and risk factors. Epidemiology Review, Oxford, v. 12, p. 1-15, 1990.

BOCCHI, E.A.; MARIN-NETO, J.A. Aplicação dos princípios da medicina baseada em evidências em programas de avaliação de procedimentos e medicamentos de alto custo. Arquivos Brasileiros de Cardiologia, São Paulo, v. 76, n.4, p. 339-342, 2001.

BOMBARDIER, C.; EISENBERG, J.M. Looking into the crystal ball: can we estimate the lifetime cost of rheumatoid arthritis? Journal of Rheumatology, Toronto, v. 12, n. 2, p. 201-204, 1985. 
BRANDLE, M. et al. Cost effectiveness of statin therapy for the primary prevention of major coronary events in individuals with type 2 diabetes. Diabetes Care, Alexandria, v. 26, n. 6, p. 1796-1801, 2003.

BRASIL. MINISTÉRIO DA SAÚDE. Caderno de Informações de Saúde. São Paulo, 2007. - Coordenação de Doenças Cardiovasculares. Doenças Cardiovasculares no Brasil. Sistema Único de Saúde - SUS. Dados Epidemiológicos e Assistência Médica. Brasília, 36 p., 1993 b. . Estatísticas de Mortalidade, 1999. Brasília, 2002a.

Fundação Nacional de Saúde, Centro Nacional de Epidemiologia. Coordenação de Informática em Saúde. Estatística de Mortalidade. 1988. Centro de Documentação. Brasília, 1993a.

Secretaria da Ciência, Tecnologia e Insumos estratégicos. Departamento de Ciência e Tecnologia. Diretrizes metodológicas: Estudos de avaliação econômica de tecnologias em saúde, Brasília, 150p., 2009.

. Secretaria Executiva. A saúde no Brasil: Estatísticas essenciais 1990-2000. 52 p.: il. - Série G. Estatística e Informação para Saúde; n. 22. Brasília, 2002b.

CESARINO, E.J. et al. Semana do coração em Ribeirão Preto - SP: resultados obtidos no período de 1993-1996. Arquivos Brasileiros de Cardiologia, São Paulo, v. 69, (suppl I) p. 121, 1997. CESARINO, E.J.; VICHI, F.L. Mortalidade hospitalar por doenças do aparelho circulatório de 1987 a 1992 em Ribeirão Preto - SP. Arquivos Brasileiros de Cardiologia, São Paulo, v. 65 (suppl. I), p. 70, 1995.

CHOR, D. et al. Doenças cardiovasculares, panorama da mortalidade no Brasil. In: MINAYO, M.C. (Org.). Os muitos brasis: saúde e população na década de 80. São Paulo: Hucitec, p. 57-86, 1995.

COLHOUN, H. et al. Collaborative Atorvastatin Diabetes Study (CARDS). American Diabetes Association, Alexandria, Jun., 2004

DATASUS. Disponível em: <http://tabnet.datasus.gov.br> Acesso em: 17 nov. 2008.

DATASUS. Disponível em: http://sigtap.datasus.gov.br/tabela-unificada/app/sec/ procedimento/publicados/consultar. Acesso em: 27 maio 2010.

DEL NERO, C.R. O que é economia da saúde. In: SF PIOLA; VIANNA, S.M. (Org.). Economia da saúde: conceitos e contribuição para a gestão da saúde, Brasília: Ipea, 1999, p. 5-21. DIÁRIO OFICIAL DA UNIÃO (D.O.U.), 27/04/10, p.45, seção 1, nº 78. Amplia a cobertura do Programa Farmácia Popular do Brasil - Aqui Tem Farmácia Popular, 2010.

DOWNS, J.R. et al. Afcaps/TexCaps Research Group Primary prevention of acute coronary events with lovastatin in men and women with average cholesterol levels: results of Afcaps/ TexCaps. Journal of the American Medical Association, Chicago, v. 279, n. 20, p. 1615-22, 1998. 
DRUMMOND, M.F. Guidelines for pharmacoeconomics studies. The ways forward. PharmacoEconomics, Bridgewater, v. 6, n. 6, p. 493-497, 1994.

FOLLADOR, W. Sobre a importância da farmacoeconomia. Saúde e Economia, São Paulo, v. 1, n. 2, p. 6-9, 1999.

FONSECA, F.A.H.; FOLLADOR, W. Farmacoeconomia e as vastatinas na doença cardiovascular. Revista Brasileira de Medicina, São Paulo, v. 60, n. 6, p. 357-363, 2003.

FREEMAN, W. M. Statins, cholesterol, and the prevention of coronary heart disease. The Federation of American Societies for Experimental Biology Journal, Bethesda, v. 20, n. 2, p. 200-1, 2006.

GAGLIARDI, T.R.A.; ROMITI, M.; XAVIER, H.T. Risco cardiovascular na mulher, São Paulo: BBS Editora, 2005. p. 147.

GILLUM, R.F. Epidemiology of heart failure in the United States (Editorial). American Heart Journal, Durham, v. 126, n. 4, p. 1042-7, 1993.

GUIA FARMACÊUTICO BRASÍNDICE, ano 45, v. 706, 418p., 2010.

HEART PROTECTION STUDY COLLABORATIVE GROUP. MRC/BHF Heart Protection Study of Cholesterol lowering with sinvastatin in 20536 high-risk individuals: a randomized placebo-controlled trial. Lancet, Londres, v. 360, n. 9326, p. 7-22, 2002.

HIPPISLWY-Cox, J.; COUPLAND, C. Effect of statins on the mortality of patients with ischaemic heart disease: population based cohort study with nested case-control analysis. Heart British Cardiac Society, Londres, v.92, n.6, p.752-8, 2006.

INSTITUTO BRASILEIRO DE GEOGRAFIA E ESTATÍSTICA. Disponível em: http://www.ibge.gov.br/home/estatistica/populacao/condicaodevida/indicadoresminimos /sinteseindicsociais2006/indic_sociais2006.pdf. Acesso em: 15 abr. 2008.

KOREN, M.J.; HUNNINGHAKE, D.B.; ALLIANCE Investigators. Clinical outcomes in managed-care patients with coronary heart disease treated aggressively in lipid-lowering disease management clinics: the alliance study. Journal of the American College.of Cardiology, San Diego, v. 44, n. 9 p. 1772-9, 2004.

LERNER, D.J.; KANNEL, W.B. Patterns of coronary heart disease morbidity and mortality in the sexes: a 26 year follow-up of the Framinghan population. American Heart Journal, Durham, v. 111, n.2; p. 383-90, 1986.

LESSA, I. Aspectos sociais da mortalidade precoce (15-59 anos) por doenças cerebrovasculares. Arquivos Neuropsiquiatria, São Paulo, v. 48, n. 3 p. 296-300, 1990.

LICHTENSTEIN, K.; ARMON, C.; BUCHACZ, K. Analysis of cardiovascular risk factors in the HIV outpatient study (HOPS) cohort. Thirteenth Conference on Retroviruses and Opportunistic Infections (CROI), Febr. 5-8, Denver, 2006. 
MARTINEZ, T.L.R. et al. Campanha Nacional de Alerta Sobre o Colesterol Elevado. Determinação do Nível de Colesterol de 81.262 Brasileiros. Arquivos Brasileiros de Cardiologia, São Paulo, v. 80, n. 6, p. 635-8, 2003.

MARTINEZ, T.L.R. Manual de condutas clinicas em dislipidemias. Rio de Janeiro: Medline, p. 35, 2003.

McMURRAY, J. et al. Trends in hospitalisation for chronic heart failure in Scotland 19801990. European. Heart. Journal, Oxford, v. 14, n. 9, p. 1158-62, 1993.

MÉDICI, A.C. Economia e financiamento do setor saúde no Brasil: balanços e perspectivas do processo de descentralização. São Paulo: Faculdade de Saúde Pública da USP, 1994.

MENNICKENT, S.C. et al. Efectos pleiotrópicos de las estatinas. Revista Médica de Chile, Concepción, v. 136, n. 6, p. 775-782, 2008.

MILLER, G.J. Lipoproteins and the haemostatic system in atherothombotic disorders. Baillière's Clinical Haematology, Londres, v. 7, n. 3, p. 713-32, 1994.

MION-JR, D.; NOBRE, F. Risco cardiovascular global: convencendo o paciente a reduzir o risco. 3 ed. São Paulo: Lemos, 2002.

MURRAY, C.J.L.; LOPEZ, A.D. Evidence-based health policy-lessons from the Global Burden of Disease Study. Science, Cambridge, v. 274, n. 5288, p. 740-3, 1996.

NATIONAL HEART, LUNG AND BLOOD ASSOCIATION. Death and age-adjusted death rates for stroke, US, 1979-2000. Morbidity \& mortality. Chart Book on Cardiovascular, Lung and Blood Diseases, Bethesda, MD: National Institutes of Health, 2002.

PARAMESHWAR, J.; POOLE-WILSON, P.A.; SUTTON, G.C. Heart failure in a district general hospital. Journal of the Royal College of Physicians of Londres, Londres, v. 26, n. 2, p. 139-42, 1992.

RIBEIRÃO PRETO (Cidade). Prefeitura Municipal. Secretaria Municipal da Saúde. Departamento de Informática. Boletim do Sistema de Coleta e Análise de Estatísticas Vitais, Ribeirão Preto, n. 4, p. 1-18, 1996.

SACKS, F.M. et al. Cholesterol and Recurrent Events Trial Investigators (CARE). The effect of pravastatin on coronary events after myocardial infarctions in patients with average cholesterol levels. The New England Journal of Medicine, Waltham, v. 355, n. 14, p. 1001-9, 1996.

SACRISTÁN DEL CASTILHO, J.A. Farmacoeconomia y evaluación económica de medicamentos: introducción. In JA Sacristán Del Castilho \& XB Llach. Farmacoeconomia: evaluación económica de medicamentos, Madrid: Médica, p. 19-29, 1995.

SCANDINAVIAN SIMVASTATIN SURVIVAL STUDY GROUP. Randomised trial of cholesterol lowering in 4.444 patients with coronary heart disease: The Scandinavian Simvastatin Survival Study (4S). Lancet, Londres, v. 344, n. 8934, p. 1383-9, 1994. 
SECOLI, S.R. et al. Farmacoeconomia: perspectiva emergente no processo de tomada de decisão. Ciência \& Saúde Coletiva, São Paulo, v. 10 (Supl.), p. 287-96, 2005.

SHEPHERD, J. et al. West of Scotland Coronary Prevention Study Group. Prevention of coronary heart disease with pravastatin in men with hypercholesterolemia. The New England Journal of Medicine, Waltham, v. 333, n. 20, p. 1301-7, 1995.

SOCIEDADE BRASILEIRA DE CARDIOLOGIA. Departamento de Aterosclerose. IV Diretriz Brasileira sobre Dislipidemias e Prevenção da Aterosclerose. Arquivos Brasileiros de Cardiologia, São Paulo, v.88 (supl 1), p. 2-19, 2007.

STEWARD, S. et al. More "malignant" than cancer? Five-year survival following a first admission for heart failure. European Journal of Heart Failure, Oxford, v. 3, n. 3, p. 315-22, 2001.

TARRAGA-LOPEZ, P. et al. A pharmacoeconomic evaluation of statins in the treatment of hypercholesterolaemia in the primary care setting in Spain. Pharmacoeconomics, Nova York, v. 23, n. 3, p. 275-87, 2005.

THE LONG-TERM INTERVENTION WITH PRAVASTATIN IN ISCHAEMIC DISEASE (LIPID) STUDY GROUP. Prevention of Cardiovascular Events and Death with Pravastatin in Patients with Coronary Heart Disease and a Broad Range of Initial Cholesterol Levels. The New England Journal of Medicine, Waltham, v. 339, p.1349-57, 1998. UNIVERSIDADE DE SÃO PAULO. Departamento de Medicina Social. Centro de Processamento de Dados Hospitalares. Internaçôes hospitalares por doenças do aparelho circulatório nos Hospitais de Ribeirão Preto - SP no ano de 1995, Ribeirão Preto, 1999, 6p.

VILLAR, F.A. Evaluación económica aplicada a los medicamentos: características y metodologia. In: JA Sacristán Del Castilho \& XB Llach. Farmacoeconomia: evaluación económica de medicamentos, Madrid: Medica, 1995, p. 31-50.

WILSON, P.W.F. et al. Prediction of coronary heart disease using risk factor categories. Circulation, Dallas, v. 97, p. 1837-47, 1998.

WOOD, D. et al. Prevention of coronary heart disease in clinical pratice. Recomendations of the Second Joint Task Force of European and other Societies on Coronary Prevention. European Heart Journal, Oxford, v. 19, p. 1434-503, 1998.

XAVIER, H.T. Manual de Dislipidemias e Cardiometabolismo, São Paulo: BBS, 2004, 215 p. YUSUF, S. et al. Effect of potentially modifiable risk factors associated with myocardial infarction in 52 countries (INTERHEART study): case-control study. Lancet, Londres, v. 364, n. 9438, p. 937-52, 2004. 


\section{Clinical and socioeconomic aspects of dyslipidemia in patients with cardiovascular diseases}

This paper discusses the clinical and socioeconomic factors arising from the presence of dyslipidemia in patients with cardiovascular disease (CVD). There are numerous studies related to CVD, oral use of statins as statins, and the economics aspects involved with an impact on health. In addition to demonstrating the importance of the treatment of dyslipidemia, the paper shows the pharmacoeconomic viewpoint, i.e. costs generated by the pharmacological treatment of this disease versus the costs of cardiovascular events and their consequences. There is therefore relevant relationship between the social impacts arising from physical disability and work, early retirements, among other important costs that could be avoided with a comprehensive economic analysis and efficient health services in Brazil. In this context, we emphasize the importance of joint analysis of the clinical and socioeconomic aspects of dyslipidemia that could influence the decisions of health authorities at the time of preparation of clinical protocols of pharmacological treatments to be implemented within the SUS.

Key words: cardiovascular diseases; dyslipidemia; statins, economics in health. 